\title{
Di-terial - Matching Digital Fabrication and Natural Grown Resources for the Development of Resource Efficient Structures
}

\author{
Felix Amtsberg ${ }^{1(\bowtie)}$, Caitlin Mueller $^{2(凶)}$, and Felix Raspall ${ }^{3(凶)}$ \\ 1 ICD, University of Stuttgart, Keplerstrasse 11, 70174 Stuttgart, Germany \\ felix.amtsbergeicd.uni-stuttgart.de \\ 2 Massachusetts Institute of Technology, 77 Massachusetts Avenue, Cambridge, \\ MA 02139, USA \\ caitlinmamit.edu \\ 3 DesignLab, Adolfo Ibanez University, 2700 Diagonal Las Torres, Santiago, Chile \\ felix.raspall@uai.cl
}

\begin{abstract}
The research presented in this paper focusses on the concept of "Diterial" which aims to merge digital design and fabrication technology with natural materials such as bamboo poles and raw timber. It proposes a digital workflow that uses sensing techniques to gain individual material information of natural, unprocessed construction resources and identify their individual strengths and characteristics and therefore its potential in load-carrying structures. This information is then used to develop bespoke designs and fabrication concepts, bridging the gap between unprocessed material and automated fabrication setups. Two case studies, developed to prove this concept, are described and compared. Both cases focused on the development of spatial structures using node-bar combinations of local resources.
\end{abstract}

Keywords: Visual sensing - 3D-printing - Robotic fabrication · Node design · Material analysis - Bamboo structures - Timber structures

\section{Context and Relevance}

Natural grown resources such as raw timber and bamboo poles are important construction materials with a long history in architecture but have been relegated in favor of the nowadays dominant materials concrete or steel. The building sector is the major contributor to climate change, accounting for over $30 \%$ of global green-house gas emissions, $40 \%$ of global energy use and $50 \%$ of global waste [1]. Due to the climate crises, the dominance of concrete and steel is being contested. Rising prices on the commodity markets make regrowing local resources an important factor for the near future in Architecture Engineering and Construction (AEC).

Currently, the construction industry is based on standardization. This determines how natural materials are processed and used: irregular logs and poles, for example, 
are usually cut into slats or strips, which are then re-joined via gluing. The resulting semi-finished goods include glue-laminated bamboo panels or timber, chipboards or LVL, among others. This standardization paradigm presents several drawbacks. First, during processing, the unique and highly efficient individual characteristics that trees and bamboos develop while growing in a specific natural environment are largely lost. Second, the processing of materials is machine- and labour-intensive and discards a significant amount of material due to offcuts. Third, the gluing results in a worse energy balance of the material. For example, Glulam, LVL, plywood and OSB used 8, 16, 19 and $28 \%$ respectively of total energy consumption for resin production [2]. Finally, the standardization of components influences, at least to some extent, the standardization of architectural concepts and may results in similar construction and building typologies.

\section{Material Scanning in AEC Industry and Research}

Scanning natural materials to identify their geometry and properties exist in the AEC industry today. The timber/sawmill industry implements high performance analysis tools like 3D scanners or computer tomography for logs. These are used to gain information of each individual piece of lumber and generate cutting plans optimize the production of construction elements according to standards and to cut out any defect like knots, holes or pitch pockets [3]. In the research community, the use of material information obtained through computer vision has been tested in a large variety of applications: it has been used to upcycle scrap material and inform robotic assembly sequences [4], to assemble small scale structures or establish material libraries of natural grown resources i.e. timber crotches [5], to identify the best fitting element and use it only processed at the connection points to build a bis scale structure for a barn. These and other examples show the immense capacity of material scanning for a smart (re) use in digital fabrication processes.

\subsection{Di-terial}

The concept "Di-terial" investigates smart design-to-fabrication systems that bring together cutting-edge digital technologies and raw, natural-grown resources. Aiming to develop highly efficient loadbearing structures in architectural scale, they use local resources. Di-terial uses material scanning as a tool to understand the individual material properties that each natural element has and take advantage of its unique qualities (Fig. 1).

\subsection{The Central Role of the Node}

This research evaluates the concept of Di-terial in the design of space frames. Space frames combine bars-linear elements- and nodes -points where bars converge- into a three-dimensional structural system. They are notable for being lightweight and extremely efficient structures. The separation of the structure into relatively small nodes and bars simplifies prefabrication in a controlled environment using technology unsuitable for onsite use and eases transportation to the construction site. Space frames can 


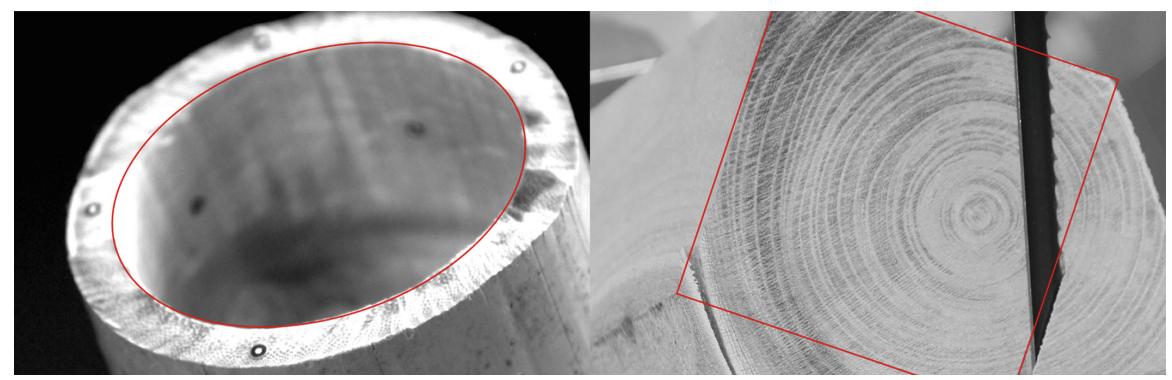

Fig. 1. Detailed individual material information investigated in this research: section geometry (bamboo) and fiber orientation (timber)

be configured to achieve structures of almost any shape, size and use, and to dis- and reassemble them for a second life cycle if needed. The node design is crucial for space frame's flexibility and efficiency. The joining concept with the bar, the valence of the system, the structural design of system and a corresponding node performance are the main questions to answer for a successful node-bar system. During the $20^{\text {th }}$ century the influence of mass- and prefabrication concepts for standardized constructions, has produced various node concepts. Above all, the developments of Konrad Wachsmann with the General Panel System for housing and the USAF Node for industrial, have undertaken the effort to enable greatest freedom possible with standard components. However, the nodal joint was too complex to be economically successful [6]. The simpler MERO-Node which was developed in 1937 and is still in use today, enables multiple configurations of space frameworks but is still limited to the geometric constraints of the node.

The digital design-to-fabrication methods of the recent years have introduced new ways for efficient production of one-off components. CNC-technology like Industrial robots have been used for custom node fabrication and the recent advancements in additive manufacturing enabled an even higher degree of customization of structural connectors [7].

\subsection{Case Studies}

Two separate case studies were developed with researchers from Singapore University of Technology and Design (SUTD) in Singapore and Massachusetts Institute of Technology (MIT) in Massachusetts using natural grown resources from the local environment (bamboo in South East Asia and timber in New England). Both projects developed customized node-bar systems based on the concept of Di-terial. It was important for this research to enable a maximum variety of design possibilities, deriving from the individual structural strengths of each material.

With a node-bar system identified as the construction system, the natural materials were used for the bars and for the node in Singapore and Cambridge, respectively. Both projects developed design-to-fabrication processes which could use the material as barely processed as possible and find their best possible fit in complex but efficient structural 
system. Digital design methods were used to produce the bespoke node geometries (Fig. 2).

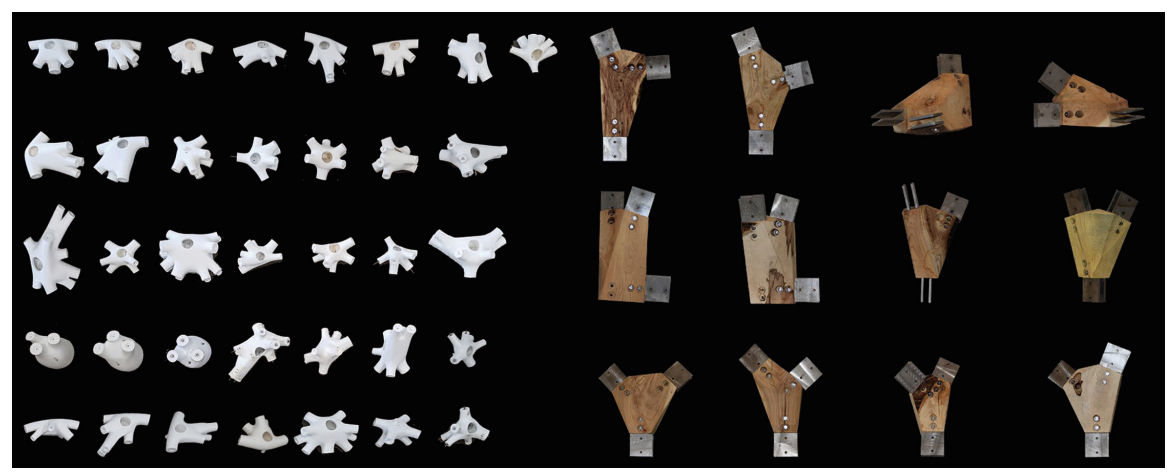

Fig. 2. 36/12 bespoke and digitally fabricated nodes merge the individual strength of natural grown resources in spatial frameworks

\subsubsection{Case Study "bamboo" 3 ",}

The project "bamboo" 3 " investigates the combination of 3D-printing technology and unprocessed bamboo. Forming hollow straight tubes by nature, bamboo poles appear to be the ideal base material for trusses and bar-node systems. A simple 2D- scanning process is used to gain precise inner and outer contour curves of the bamboo section, which was then used to inform the geometry of the bespoke connectors (Fig. 3).
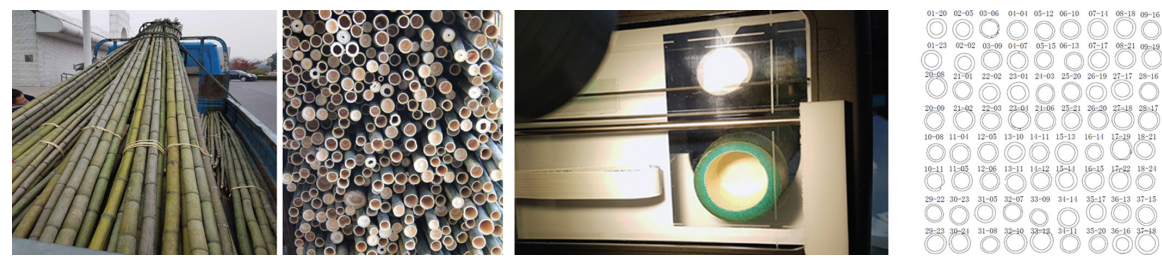

Fig. 3. Collected bamboo, scanning of the section geometry and digital representation

As mentioned before, 3D-printing enables the fabrication of almost any shape, but especially the chosen method FDM-printing of biodegradable PLA, results in limited structural performance. Thus, it was decided to focus on structural systems, where the node only must react to normal forces but not bending moments. Examples (Fig. 4) are trussed beam structures (a) and triangulated grid shells (b), but also spatial grids like tetrahedral meshes (c), which was chosen for the final design (d). These systems, especially if irregular request an efficient fabrication of one-off node due to unique angle combinations and various node valences in case of the realized structure 3 to 9 . 


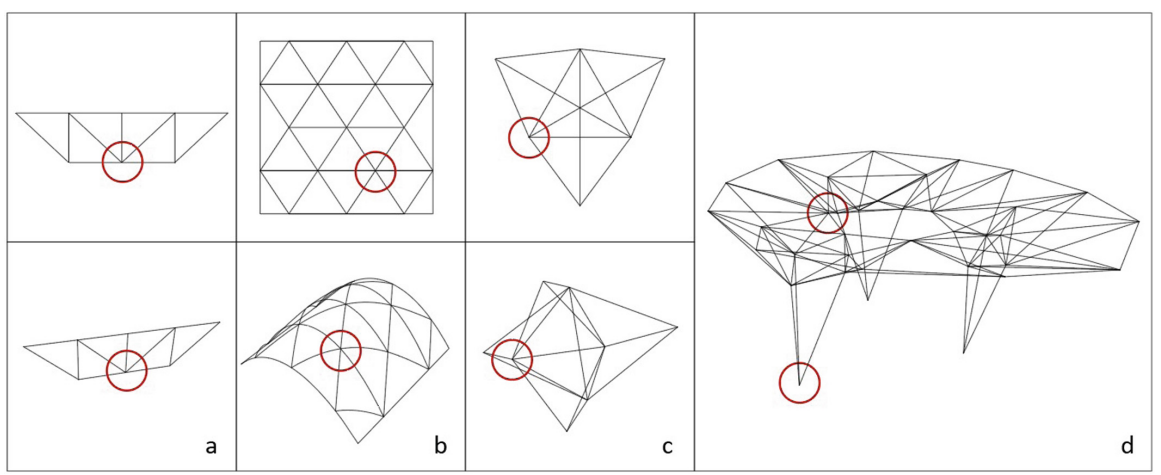

Fig. 4. Spatial structures in $2 \mathrm{D}$ (a: trussed beam/valence $2-5$, b: triangulated grid shell/valence 2-3) and 3D (c: tetrahedral mesh/valence 2-n) were investigated and led to the final design (d: Sombra Verde/valence 3-9)

A Grasshopper@ script using Karamba@ [8] was written to compare load carrying capacity of the bamboo pole and structural requirements of the design to identify the bamboo thickness (Fig. 5a). The results were categorized in bamboo diameter $>30,40$ and $50 \mathrm{~mm}$ and used to inform the node diameter at the node-bar connection (b). Combined with the information of mesh angles and valence, this input is used to automatically generate the node design (c) and print (d) the nodes.

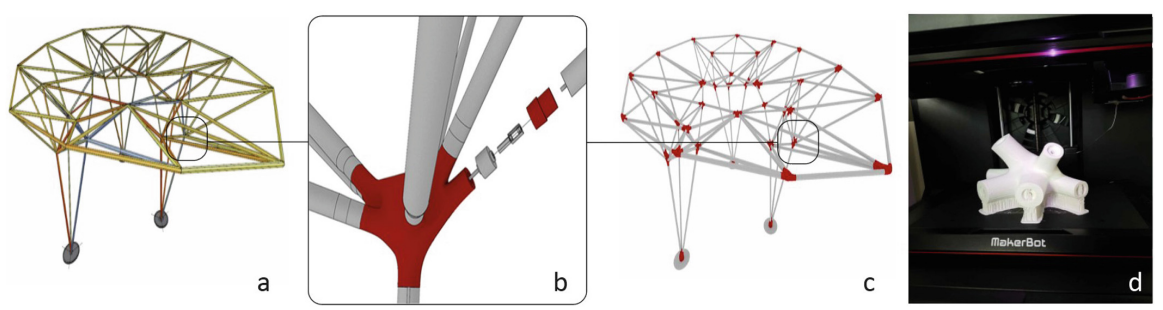

Fig. 5. Structural analysis of the designed bamboo gazebo (a) led to the node and dowel (red) geometries (b), their implementation in the structure (c) and production via 3D-printing of a node

Conventional PLA based 3D-printers were used to produce the 238 unique connectors and 36 nodes that joined the 117 bamboo poles. The fabrication time varied between 21 and $128 \mathrm{~h}$ for the node elements and 1.5 to $4 \mathrm{~h}$ for the dowels, respectively. These elements formed "Sombra Verde" a shade providing structure in Duxton Plain park Singapore. The 3-legged, 6 by $8 \mathrm{~m}$ spanning structure was installed for 3 months during the "Urban Design week 2018" (Fig. 6).

\subsubsection{Case Study "Structural Upcycling”}

The project "Structural Upcycling" investigates the use of robotically subtractive fabricated timber crotches. The typically Y-shaped crotches of deciduous trees form natural 

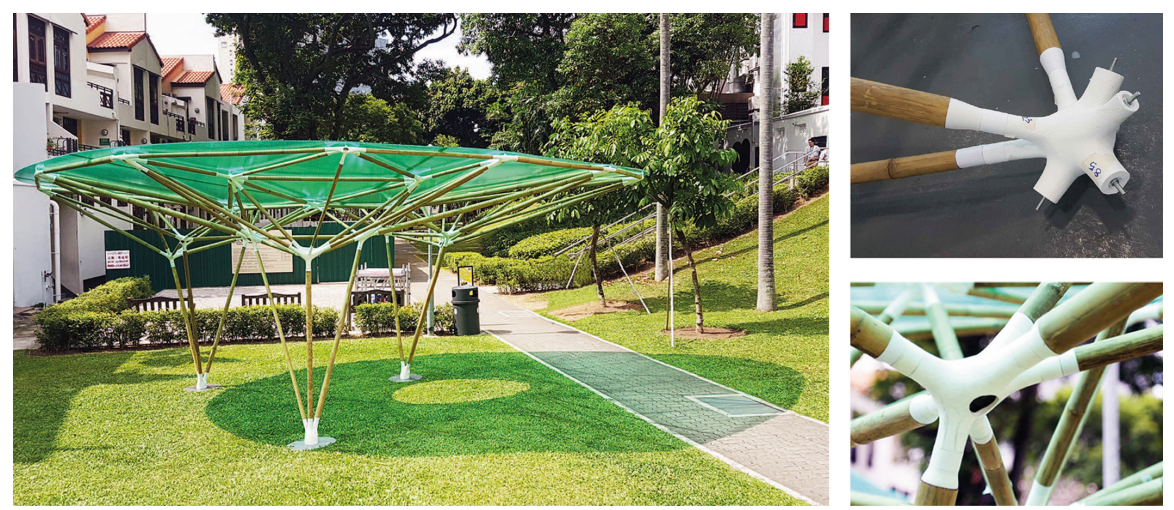

Fig. 6. Installed bamboo gazebo "Sombra Verde" and node close-ups

cantilevers and present a complex fiber orientation. For this reason, they are usually discarded during the fabrication of standard timber products despite their structural potential in structures with similar structural requirements as the original crotches [9]. This case study takes advantage of this natural structural design, using the crotches as bending stiff nodes in node-bar systems.

Low-cost consumer-grade 3D-Scanners are used to generate a 3D-mesh of the crotch surface. The meshes are simplified and the original branch vectors determined. A material library, an inventory of scanned crotches, is established and used to match a developed design concept (Fig. 7).
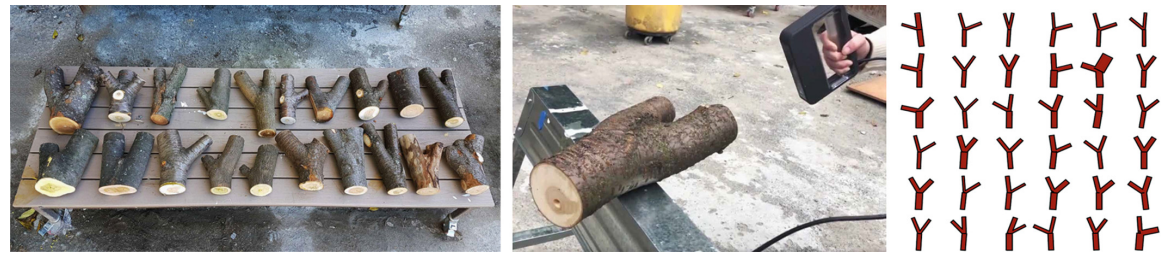

Fig. 7. Sourced crotches are 3D-scanned to establish the material library

Since the design system is based on the use of predominately 3-valence bending nodes crotches (Fig. 8) honeycomb meshes (a), more patterns like Voronoi (c) or certain Archimedean solids were investigated to establish the final design (d).

It is also essential for the morphology of the nodes in the structure to match the branch vectors as closely as possible, to benefit from the fiber orientations in the crutches. Large mismatches between designed structural node and the assigned crutch result in a decrease of the structural performance of the node [10]. Therefore, a matching algorithm was developed to assign the best-fitting crutch to each node in the structure and adapt the structure's morphology to the inventory of available crutches (Fig. 9 a). This matching concept has been explained in a separate publication [11]. In theory, the crotch achieves the best structural performance unprocessed, when no fibers are cut. However, this results 


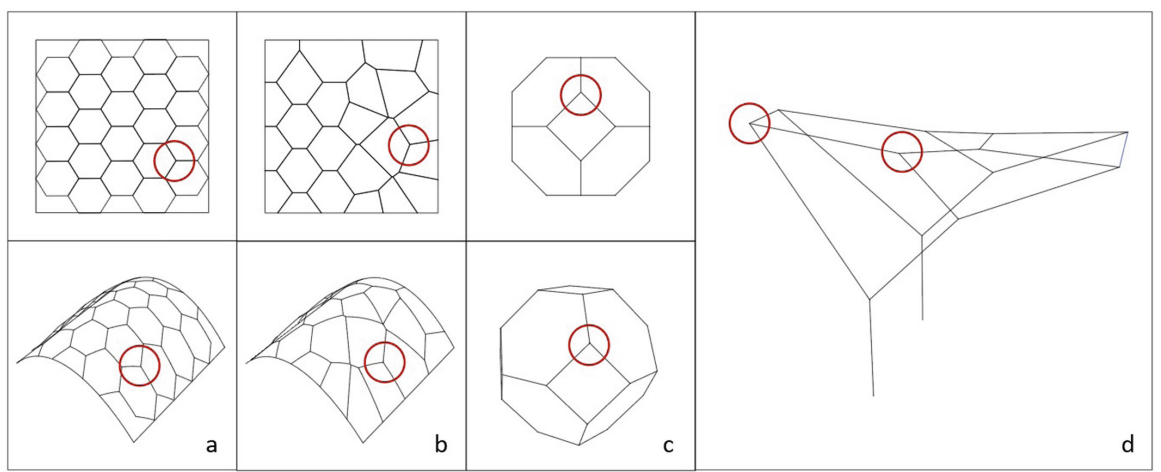

Fig. 8. Spatial structures in 2D (a: hexagonal grid shell, b: Voronoi) and 3D (c: truncated Icosahedra) were investigated and led to the final design (d)

in several disadvantages, when the unprocessed nodes are joined with the standard linear elements. Therefore, the node design was developed based on the logic of a convex hull (Fig. 9b,c), a geometry which represent the smallest convex structure to cover the contact faces and generates a triangulated node. The convex hull has two key benefits: it creates a geometry that is compatible with the standard lumber sections of the bars converging in the node, and it creates a geometry without valleys, which can be manufactured using simple planar cuts with a band saw. This case study takes advantage of this natural structural design, using the crotches as bending stiff nodes in node-bar systems.

A second algorithm generates a node geometry which fills the scanned crotch mesh and expands its for to maximize the use of material available in the crutch. The rectangular contact faces fit the corresponding bars and establish a well-balanced compromise between maximized material use, minimal fabrication time and matches one-off and standard components. For the fabrication, a robotic work cell was developed, in which an industrial robot holding a crutch was located in front of a standard bandsaw (d). The script automatically generates the cutting sequence, resulting in a time-saving production of one-off component with just a few straight cuts (between 12 and 25 in the case study). Thus, the fabrication time of the convex hull took between 13 and 35 min depending on dimension of the node and the number of faces, cutting depth and hardness.

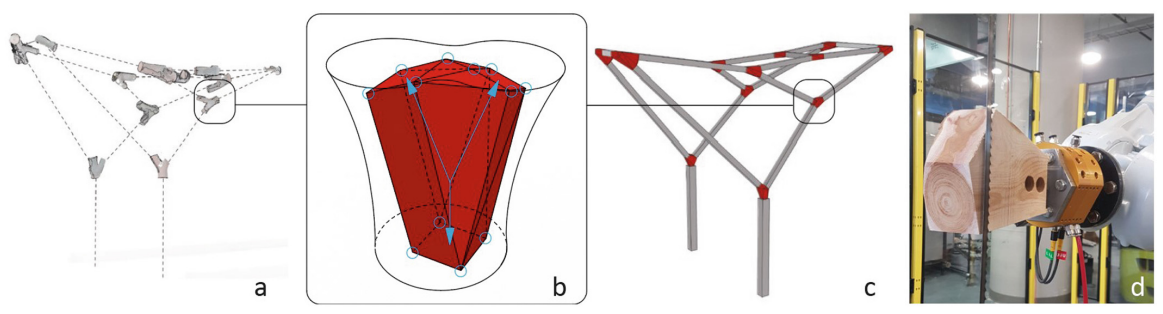

Fig. 9. Matching crotches were placed in the designed mock-up (a) a convex node geometry (b), their implementation in the structure (c) and production via robotic band sawing of a node 
A full-scale mock-up was developed and installed at the MIT School of Architecture and Planning. The prototype demonstrated that the workflow, from scanning of crutches to node generation and allocation in the structure, to the robotic fabrication and assembly, are fully functional. The results of the mock-up also served as preparation for a permanent structure which is currently in the planning phase. The crotches were sourced from trees that had been felled during a renovation process in an urban environment. The pieces not collected by the researcher had no value and were chipped immediately. 12 crotches were processed and joined with $191 \frac{1 / 2}{2} \times 1 \frac{1}{2}$ inch bars to form this $4 \mathrm{~m} \times 2 \mathrm{~m}$ prototype (Fig. 10).
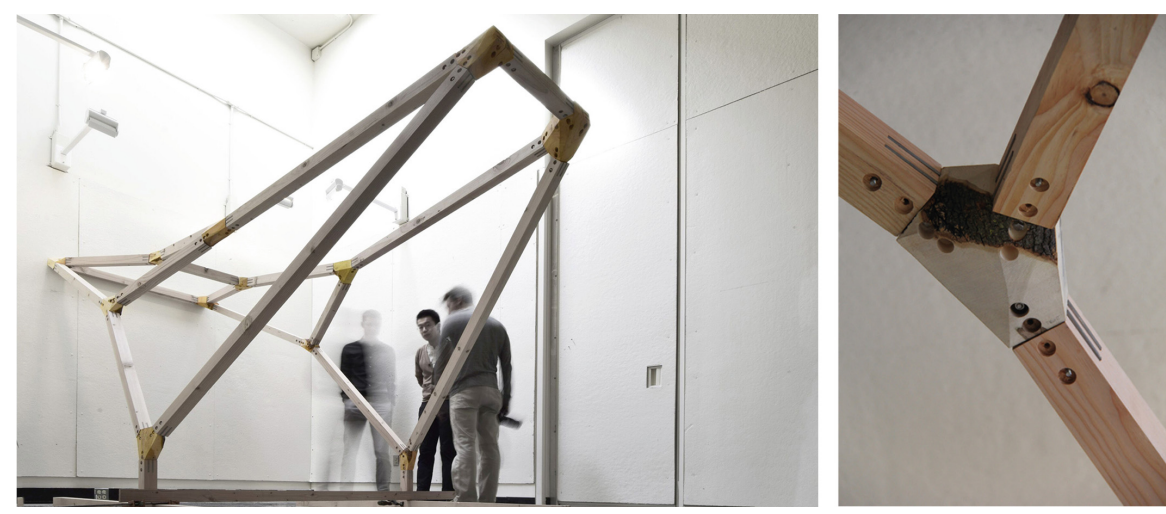

Fig. 10. Exhibition of the mock-up to test the case study "Structural Upcycling"

\section{Comparison, Conclusion and Outlook}

As described, both case studies focused on specific implementations of Di-terial, but, in retrospect, they would significantly benefit from each other's innovations.

Bamboo $^{3}$ : The creation of an inventory of all scanned bamboo poles was used solely to create bespoke connectors. However, this information could be used for alternative strategies such as optimizing the structure based on the actual moments of inertia or cutting longer poles into shorter pieces. Additionally, the node printing orientation was determined to minimize printing speed, but could have been optimized for mechanical strength.

Structural Upcycling: Due to time constraints, the bar elements in the project had a fixed section. Applying different section geometries would result in a higher efficiency of the material use. The algorithm developed to generate the nodes already includes this functionality. Additional information like material hardness could generate more effective libraries. Alternative geometries beyond the convex hull could exploit more efficient material performance. Additional studies investigated the matching potential 
of different tree species for designed geometries, based on their typical ratio of branch angles [12].

The projects presented in this paper, show two different, successful approaches to Diterial. They analyze and identify individual strengths of natural grown local resources using material sensing. A Structural system, which takes advantage of these specific strengths is defined, designed and structural analysis software calculates the designed structure. That way, the Material placement happens were needed. Digital Fabrication Concepts are developed to join natural-grown construction materials seamlessly in a well-balanced resource efficient system.

One of the major challenges of the 21 st century is the design, planning and construction of sustainable building and structures. Natural-grown resources show an immense capacity for application in digital fabrication and sustainable architecture. As data acquisition, storage and processing become increasingly affordable and ubiquitous, new digital workflows such as those described in this paper, can be developed to take advantage of non-standard materials with more complex structures. In this way, individual characteristics of each naturally grown material component are computationally analyzed and its features considered an opportunity rather than a disadvantage.

Acknowledgements. We would like to thank our sponsors, contributors and researchers:

Bamboo $^{3}$ : Urban Redevelopment Authority and Lope Lab, AIRLab, Yuxin Hu, Sourabh Maheshwari, Jenn Chong, Anna Toh, Aurelia Chan, and Sihan Wu.

Strucutral Upcycling: MIT School of Architecture Woodshop, Autodesk Build Space Boston, Yijiang Huang, Kevin Moreno Gata, Daniel M. Marshall.

\section{References}

1. United-Nations. Buildings and climate change: summary for decision makers (2009). https:// www.unglobalcompact.org/

2. Puettmann, M., Wilson, J.: Life-cycle analysis of wood products: cradle-to-gate LCI of residential wood building materials. Wood Fiber Sci.: J. Soc. Wood Sci. Technol. 37, 18-29 (2005)

3. Rais, A., Ursella, E., Vicario, E., Giudiceandrea, F.: The use of the first industrial X-ray CT scanner increases the lumber recovery value: case study on visually strength-graded douglas-fir timber. Ann. For. Sci. 74(2), 28 (2017)

4. Raspall, F.: Design with material uncertainty: responsive design and fabrication in architecture. In: Thomsen, M.R., Tamke, M., Gengnagel, C., Faircloth, B., Scheurer, F. (eds.) Modelling Behaviour, pp. 315-327. Springer, Cham (2015). https://doi.org/10.1007/978-3319-24208-8_27

5. Self, M., Vercruysse, E.: Infinite variations, radical strategies. In: Fabricate 2017 Conference Proceedings, pp. 30-35. UCL Press, London (2017). JSTOR

6. Barthel, R., Junge, R., Krippner, R., Petzold, F.: Wendepunkte im Bauen: Von der seriellen zur digitalen Architektur. In: Wendepunkte im Bauen. Detail (2010)

7. Bañón, C., Raspall, F.: 3D Printing Architecture: Workflows, Applications, and Trends. Springer, Singapore (2020). https://doi.org/10.1007/978-981-15-8388-9 
8. Karamba3D (2021). Karamba3d: a parametric structural engineering tool. https://www.kar amba3d.com/

9. Mattheck, C.: Design in Nature Learning from Trees. Springer Berlin (1998)

10. Hankinson, R.: Investigation of crushing strength of spruce at varying angles of grain. Air Serv. Inf. Circular 3(259), 130 (1921)

11. Amtsberg, F., Huang, Y., Marshall, D., Moreno, K., Mueller, C., et al.: Structural upcycling: matching digital and natural geometry in AAG 2020 (in print, 2021)

12. Desai, I.: Designing structures with tree forks: mechanical characterization and generalized computational design approach. Master's thesis, Massachusetts Institute of Technology (2020)

Open Access This chapter is licensed under the terms of the Creative Commons Attribution 4.0 International License (http://creativecommons.org/licenses/by/4.0/), which permits use, sharing, adaptation, distribution and reproduction in any medium or format, as long as you give appropriate credit to the original author(s) and the source, provide a link to the Creative Commons license and indicate if changes were made.

The images or other third party material in this chapter are included in the chapter's Creative Commons license, unless indicated otherwise in a credit line to the material. If material is not included in the chapter's Creative Commons license and your intended use is not permitted by statutory regulation or exceeds the permitted use, you will need to obtain permission directly from the copyright holder.

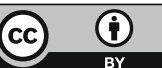

